\title{
A FREE BOUNDARY PROBLEM ARISING IN A MODEL OF WOUND HEALING
}

\author{
XINFU CHEN* AND AVNER FRIEDMAN** \\ *Department of Mathematics, University of Pittsburgh, Pittsburgh, PA 15260. \\ ${ }^{* *}$ Department of Mathematics, University of Minnesota, 206 Church St. SE, Minneapolis, MN 55455.
}

\begin{abstract}
In this paper we consider a system of two semilinear parabolic reaction-diffusion equations with free boundary, which arises in a model of corneal epithelial wound healing. We prove that the initial-boundary value problem has a unique solution and that complete healing in achieved in finite time. We then proceed to consider travelling wave solutions of the same system, and establish the existence of such a solution.
\end{abstract}

Key words. free boundary, reaction-diffusion equations, travelling wave solutions, wound healing.

AMS subject classifications. 35K57, 35R35, 92C50.

\section{Introduction.}

The mathematical modeling of wound healing has received increased attention in recent years. One area of investigation is dermal wound healing, where complex biological processes are interacting $[4,7]$. Two of these processes are the invasion of fibroblasts into the wound space and their alignment there [5], and the sprouting of blood vessel into the wound space (angiogenesis) [6]. Another area of investigation is corneal epithelial wound healing, which was modelled by Dale, Maini and Sheratt [1]. The model involves a system of two parabolic partial differential equations (semilinear reaction-diffusion equations) for the generic corneal stimulus concentration $n$, in the healed region, and the chemical stimulus concentration $c$ (also called the epidermal growth factor), both in the healed and the wound regions. Their model is 1dimensional and exhibits, after a short time, a travelling wave character. They computed the speed of the travelling wave to be approximately $20 \mu \mathrm{mh}^{-1}$; the actual observed healing rate in corneal wounds is approximately $60 \mu \mathrm{mh}^{-1}$.

This model was recently improved by Gaffney, Maini, McCaig, Zhao and Forrester [2] in two ways. First they include the presence of physiological electric field and, second, they introduce a free boundary into the model. The free boundary is the receding boundary of the wound region. Their computations show that the average speed of the free boundary varies linearly with the electric field over a large range, and that this linear relation is robust under variations of some parameters which are difficult to estimate precisely.

The purpose of this paper is to study the model introduced in [2] by rigorous mathematical analysis. In Part I we consider the semilinear reaction-diffusion system with free boundary and prove that the initial-boundary value problem has a unique solution for a time interval $0 \leq t<T_{*}$, where $T_{*}$, a finite number, is the time at which the wound becomes completely healed. In Part II we consider the travelling wave version of the model and prove that a travelling wave solution exists.

In order to make the paper more readable, we shall deal with a general system of reactiondiffusion equations, subject to some assumptions. In the concluding section of the paper we shall write down the explicit system developed in [2], and show how it satisfies the general assumptions made throughout Parts I and II. 


\section{Part I. The evolution problem}

\section{The model.}

Consider the following system for $n(x, t), c(x, t), s(t)$ :

$$
\begin{aligned}
& n_{t}=\left(d(n, c) n_{x}\right)_{x}+f(n, c), \quad 0<x<s(t), t>0, \\
& a c_{t}=c_{x x}+g(n, c), \quad 0<x<1, t>0, \quad(a \text { a positive constant }) \\
& n \equiv 0 \quad \text { if } \quad s(t)<x<1, t>0, \\
& n(s(t), t)=n_{*}, \quad t>0, \quad\left(n_{*} \text { constant }\right) \\
& \dot{s}(t)=-\frac{1}{n_{*}} d\left(n_{*}, c\right) n_{x} \quad \text { at } \quad x=s(t), t>0,
\end{aligned}
$$

with boundary conditions

$$
n_{x}(0, t)=0 \quad c_{x}(0, t)=0 \quad c_{x}(1, t)=0 \quad \text { for } \quad t>0
$$

and initial conditions

$$
\begin{array}{ll}
s(0)=s_{0}, & 0<s_{0}<1, \\
n(x, 0)=n_{0}(x), & 0<x<s_{0}, \\
c(x, t)=c_{0}(x), & 0<x<1 .
\end{array}
$$

Here $n$ represents the corneal stimulus cell density outside the wound, and $c$ represents the chemical stimulus concentration in both the wounded and healed parts.

We assume that

$$
0<n_{*}<1
$$

and set

$$
G=\left\{(n, c) ; \quad n^{*} \leq n \leq 2, \quad 0 \leq c \leq 1\right\} .
$$

We also make the following assumptions:

$$
\begin{gathered}
d(n, c) \text { is in } C^{1}(G) \quad \text { and } \partial d / \partial n \leq 0 \\
d(n, c) \geq d_{0}>0 \quad\left(d_{0} \text { constant }\right) \\
f(n, c) \text { is in } C^{1}(G) \text { and } \\
f\left(n_{*}, c\right)>0, \quad f(2, c) \leq 0 \text { if } 0 \leq c \leq 1 ; \\
g(n, c) \text { is in } C^{1}(G) \text { and } \partial g / \partial c<0 \text { in } G, \\
g(n, 0) \geq 0, \quad g(n, 1) \leq 0 \text { for } n_{*} \leq n \leq 2 ; \\
n_{0} \in C^{2}\left[0, s_{0}\right], \quad n_{*}<n_{0}(x)<2 \text { if } 0 \leq x<s_{0}, \\
n_{0}^{\prime}(0)=0, \quad n_{0}\left(s_{0}\right)=n_{*}, \quad n_{0}^{\prime}\left(s_{0}\right)<0 ; \\
c_{0} \in C^{2}[0,1] \quad \text { and } 0 \leq c_{0}(x) \leq 1 .
\end{gathered}
$$

We may clearly extend the definitions of the functions $d, f, g$ in such a way that

(1.9), (1.10), (1.11) hold for $-\infty<n, c<\infty$, and

$$
\begin{aligned}
& f(n, c)>0 \quad \text { if } \quad n \leq n_{*}, \quad f(n, c) \leq 0 \quad \text { if } \quad n \geq 2, \quad \text { for all } c, \\
& g(n, c) \geq 0 \quad \text { if } \quad c \leq 0, \quad g(n, c) \leq 0 \quad \text { if } \quad c \geq 1, \quad \text { for all } n .
\end{aligned}
$$


In the future we shall assume such an extension has already been made.

In Sections 2, 3 we shall prove the following theorem:

THEOREM 1.1. Assume that (1.8)-(1.13) hold. Then there exists a unique solution $(n, c, s)$ of (1.1)-(1.7) for $0 \leq t<T_{*}$, where $T_{*}<\infty$, and

$$
\begin{array}{ll}
s^{\prime}(t)>0 & \text { if } \quad 0 \leq t<T_{*} \\
s(t) \rightarrow 1 & \text { if } \quad t \rightarrow T_{*}
\end{array}
$$

furthermore,

$$
\begin{array}{cl}
n_{*}<n(x, t) \leq 2 & \text { if } \quad 0 \leq x<s(t), \quad 0 \leq t<T_{*} \\
0 \leq c(x, t) \leq 1 & \text { if } \quad 0 \leq x \leq 1, \quad 0 \leq t<T_{*}
\end{array}
$$

Note that (1.16) means that the wound region $\{s(t) \leq x \leq 1\}$ disappears as $t \rightarrow T_{*}$.

In $\S 2$ we shall prove local existence and uniqueness. In $\S 3$ we shall derive a priori estimates which will enable us to complete the proof of Theorem 1.1.

2. Local existence and uniqueness. In this section we prove:

LEMmA 2.1. Under the assumptions of Theorem 1.1 there exists a unique solution of (1.1)-(1.7) for a small time interval $0 \leq t \leq T(T>0)$.

Proof. Set

$$
\delta=\min \left\{s_{0}, 1-s_{0}\right\}
$$

and let $\zeta(y)$ be a function in $C^{3}[0,1]$ satisfying:

$$
\zeta(y)=1 \quad \text { if } \quad\left|y-s_{0}\right|<\frac{\delta}{4}, \quad \zeta(y)=0 \quad \text { if } \quad\left|y-s_{0}\right|>\delta, \quad\left|\zeta^{\prime}(y)\right|<\frac{2}{\delta}
$$

We introduce a transformation that will straighten the free boundary:

$$
(x, t) \rightarrow(y, t) \quad \text { where } \quad x=y+\zeta(y)\left(s(t)-s_{0}\right), \quad 0 \leq y \leq 1 .
$$

Notice that as long as

$$
\left|s(t)-s_{0}\right|<\frac{\delta}{4}
$$

the transformation $(2.1)$ is diffeomorphism from $[0,1]$ onto $[0,1]\left(\right.$ since $\left.\partial x / \partial y>\frac{1}{2}\right)$, and

$$
\begin{aligned}
0 \leq x \leq s(t) & \Longleftrightarrow 0 \leq y \leq s_{0}, \\
s(t) \leq x \leq 1 & \Longleftrightarrow s_{0} \leq y \leq 1, \\
x=s(t) & \Longleftrightarrow y=s_{0} .
\end{aligned}
$$

One easily computes that

$$
\begin{aligned}
& \frac{\partial y}{\partial x}=\frac{1}{1+\zeta^{\prime}(y)\left(s(t)-s_{0}\right)} \equiv \sqrt{A(s(t), y)}, \\
& \frac{\partial^{2} y}{\partial x^{2}}=\frac{\zeta^{\prime \prime}(y)\left(s(t)-s_{0}\right)}{\left[1+\zeta^{\prime}(y)\left(s(t)-s_{0}\right)\right]^{3}} \equiv B(s(t), y), \\
& -\frac{1}{\dot{s}(t)} \frac{\partial y}{\partial t}=\frac{\zeta(y)}{1+\zeta^{\prime}(y)\left(s(t)-s_{0}\right)} \equiv C(s(t), y) .
\end{aligned}
$$




\section{Defining}

$$
\varphi(y, t)=n(x, t), \quad \psi(y, t)=c(x, t)
$$

and setting

$$
D(\varphi, \psi)=d(n, c), \quad F(\varphi, \psi)=f(n, c), \quad G(\varphi, \psi)=g(n, c)
$$

the system (1.1)-(1.7) takes the form

$$
\begin{aligned}
& \varphi_{t}=A D(\varphi, \psi) \varphi_{y y}+(B D(\varphi, \psi)+\dot{s} C) \varphi_{y}+A D_{\varphi} \varphi_{y}^{2}+A D_{\psi} \varphi_{y} \psi_{y} \text { for } 0<y<s_{0}, \quad t>0 \\
& a \psi_{t}=A \psi_{y y}+(B+a \dot{s} C) \psi_{y}+G(\varphi, \psi) \text { for } 0<y<1, \quad t>0 \\
& \varphi \equiv 0 \text { if } s_{0}<y<1
\end{aligned}
$$

with $A=A(s, y), B=B(s, y), C=C(s, y)$,

$$
\begin{aligned}
& \varphi\left(s_{0}, t\right)=n_{*}, \\
& \dot{s}(t)=-\frac{1}{n_{*}} D\left(n_{*}, \psi\left(s_{0}, t\right)\right) \varphi_{y}\left(s_{0}, t\right),
\end{aligned}
$$

and

$$
\begin{aligned}
& \varphi_{y}(0, t)=\psi_{y}(0, t)=\psi_{y}(1, t)=0, \\
& \begin{cases}\varphi(y, 0)=\varphi_{0}(y), & 0 \leq y<s_{0} \\
\psi(y, 0)=\psi_{0}(y), & 0 \leq y \leq 1,\end{cases}
\end{aligned}
$$

where $\varphi_{0}(y)=n_{0}(x), \psi_{0}(y)=c_{0}(x)$.

We introduce the quantity

$$
s_{1}=-\frac{1}{n_{*}} D\left(n_{*}, \psi_{0}\left(s_{0}\right)\right) \varphi_{0}^{\prime}\left(s_{0}\right) \quad\left(s_{1}>0\right)
$$

which should be the derivative $\dot{s}(0)$ if a solution exists.

We shall prove existence by invoking the Schauder fixed point theorem. Toward this purpose we introduce spaces

$$
\begin{aligned}
X_{T} & =\left\{s \in C^{1}[0, T], \quad s(0)=0, \quad \dot{s}(0)=s_{1}, \quad\left|\dot{s}(t)-s_{1}\right| \leq 1 \quad \text { for } \quad 0 \leq t \leq T\right\} \\
Y_{T} & =\left\{\varphi \in C^{0}\left(\left[0, s_{0}\right] \times[0, T]\right), \quad \varphi(y, 0)=\varphi_{0}, \quad\left|\varphi-\varphi_{0}\right|_{\left.C^{0}\left(\left[0, s_{0}\right] \times[0, T]\right) \leq 1\right\}}\right.
\end{aligned}
$$

where $T$ is such that

$$
0<T<\frac{\delta}{4\left(1+s_{1}\right)} .
$$

For any $(\tilde{s}, \tilde{\varphi}) \in X_{T} \times Y_{T}$ we then have

$$
\left|\tilde{s}(t)-s_{0}\right|<\frac{\delta}{4}
$$

so that the mapping $(x, y) \rightarrow(y, t)$ defined by

$$
x=y+\zeta(y)\left(\tilde{s}(t)-s_{0}\right)
$$


is a diffeomorphism, and we define $\psi$ to be the solution of

$$
a \psi_{t}=A(\tilde{s}, y) \psi_{y y}+(B(\tilde{s}, y)+a \dot{\tilde{s}} C(\tilde{s}, y)) \psi_{y}+G(\tilde{\varphi}, \psi) \quad \text { for } \quad 0<y<1,0<t<T
$$

with $\tilde{\varphi} \equiv 0$ in $\left[s_{0}, 1\right] \times[0, T]$,

$$
\begin{aligned}
\psi_{x}(0, t) & =\psi_{x}(1, t)=0, \quad 0<t<T, \\
\psi(y, 0) & =\psi_{0}(y) .
\end{aligned}
$$

Using $L^{p}$ estimates for parabolic equations and Sobolev's inequalities, one can show that this system has a unique solution $\psi$ with finite norm

$$
\|\psi\|_{C^{1+\beta,(1+\beta) / 2([0,1] \times[0, T])}} \leq K
$$

for any $0<\beta<1$, where $K$ will be used to denote constants depending only on $s_{0}, s_{1}$, and on

$$
\left|\varphi_{0}\right|_{C^{2}\left[0, s_{0}\right]},\left|\varphi_{0}\right|_{C^{2}[0,1]} \cdot
$$

In fact, results of this type, for more general nonlinear parabolic equations, are proved in [3].

Next we define a function $\varphi$ as the solution of the parabolic problem:

$$
\begin{aligned}
& \varphi_{t}=A(\tilde{s}, y) D(\tilde{\varphi}, \psi) \varphi_{y y}+[B(\tilde{s}, y) D(\tilde{\varphi}, \psi)+\dot{\tilde{s}} C(\tilde{s}, y)] \varphi_{y}+A(\tilde{s}, y) D_{\varphi}(\tilde{\varphi}, \psi) \varphi_{y}^{2} \\
& \quad+A(\tilde{s}, y) D_{\psi}(\tilde{\varphi}, \psi) \varphi_{y} \psi_{y}+f(\varphi, \psi) \text { for } 0 \leq y \leq s_{0}, 0 \leq t \leq T
\end{aligned}
$$

As before, this system has a unique solution $\varphi$ with finite norm

$$
\|\varphi\|_{C^{1+\beta,(1+\beta) / 2}\left(\left[0, s_{0}\right] \times[0, T]\right)} \leq K .
$$

Finally we define

$$
s(t)=s_{0}-\int_{0}^{t} \frac{1}{n_{*}} D\left(n_{*}, \psi\left(s_{0}, \tau\right)\right) \varphi_{y}\left(s_{0}, \tau\right) d \tau
$$

and introduce the mapping $W$ by

$$
(s, \varphi)=W(\tilde{s}, \tilde{\varphi}) .
$$

We want to show that $W$ has a fixed point $(s, \varphi)$, which will then imply that, together with the corresponding $\psi$, it forms a solution to the system (2.3)-(2.9).

Observe that

$$
\left.\dot{s}(t)-s_{1}=\frac{1}{n_{*}}\left\{D\left(n_{*}, \psi_{0}\left(s_{0}\right)\right) \frac{\partial \varphi_{0}\left(s_{0}\right)}{\partial y}-D\left(n_{*}, \psi\left(s_{0}, t\right)\right) \varphi_{y}\left(s_{0}, t\right)\right)\right\}
$$

and the right-hand side is in $C^{\beta / 2}[0, T]$. Hence

$$
\left\|\dot{s}-s_{1}\right\|_{C^{\beta / 2}[0, T]} \leq K .
$$

By (2.12) we also have

$$
\left\|\varphi-\varphi_{0}\right\|_{C^{1+\beta,(1+\beta) / 2}\left(\left[0, s_{0}\right] \times[0, T]\right)} \leq K .
$$


Hence, if $T$ is small enough,

$$
\begin{aligned}
\left\|\dot{s}-s_{1}\right\|_{C^{0}[0, T]} & \leq\left\|\dot{s}-s_{1}\right\|_{C^{\beta / 2}[0, T]} T^{\beta / 2} \leq 1 \\
\left\|\varphi-\varphi_{0}\right\|_{C^{0}\left[0, s_{0}\right]} & \leq\left\|\varphi-\varphi_{0}\right\|_{C^{0,(1+\beta) / 2}\left(\left[0, s_{0}\right] \times[0, T]\right)} T^{\frac{1+\beta}{2}} \leq 1,
\end{aligned}
$$

so that $W$ maps $X_{T} \times Y_{T}$ into itself.

From the estimates above it follows that the image of $W$ lies in a compact subset of $X_{T} \times Y_{T}$, and a standard argument then also shows that $W$ is continuous. Invoking the Schauder fixed point theorem we conclude that $W$ has a fixed point in $X_{T} \times Y_{T}$.

We can further use the Schauder estimates to obtain additional regularity of the solution, such as the Hölder continuity of $\dot{s}(t)$, and of the second spatial derivatives of $\varphi$ and $\psi$. It remains to prove uniqueness.

Let $\left(s_{i}, \varphi_{i}, \psi_{i}\right)(i=1,2)$ be two solutions. Then

$$
\left\|s_{i}\right\|_{C^{1+\beta / 2}[0, T]}+\left\|\varphi_{i}\right\|_{C^{1+\beta,(1+\beta) / 2}\left(\left[0, s_{0}\right] \times[0, T]\right)}+\left\|\psi_{i}\right\|_{C^{1+\beta,(1+\beta) / 2}([0,1] \times[0, T])} \leq K .
$$

Setting $\psi=\psi_{1}-\psi_{2}$ and taking the difference of the equations for $\psi_{1}, \psi_{2}$, we get

$$
\begin{aligned}
a \psi_{t}= & A\left(s_{2}, y\right) \psi_{y y}+\left[B\left(s_{2}, y\right)+a \dot{s}_{2} C\left(s_{2}, y\right)\right] \psi_{y}+G_{1} \varphi+G_{2} \psi \\
& +\left[A\left(s_{1}, y\right)-A\left(s_{2}, y\right)\right] \psi_{1, y y}+\left[B\left(s_{1}, y\right)-B\left(s_{2}, y\right)\right] \psi_{1, y}+a\left[\dot{s}_{1} C\left(s_{1}, y\right)-\dot{s}_{2} C\left(s_{2}, y\right)\right] \psi_{1, y}
\end{aligned}
$$

where $G_{1}, G_{2}$ are the partial derivatives of $G$ with respect to the first and second variables evaluated at an intermediate point.

Using $W^{2, p}$ estimates for parabolic equations of the form $u_{t}-a u_{y y}-b u_{y}+c u=g$ and Sobolev's imbedding, we get

$$
\left\|\psi_{1}-\psi_{2}\right\|_{C^{1+\beta,(1+\beta) / 2}} \leq K\left\{\left\|\varphi_{1}-\varphi_{2}\right\|_{C^{0}}+\left\|s_{1}-s_{2}\right\|_{C^{1}}\right\}
$$

Similarly we derive the inequality

$$
\left\|\varphi_{1}-\varphi_{2}\right\|_{C^{1+\beta,(1+\beta) / 2}} \leq K\left\{|| \psi_{1}-\psi_{2}\left\|_{C^{1,0}}+\right\| s_{1}-s_{2} \|_{C^{1}}\right\} .
$$

Furthermore, taking the difference of the equations for $s_{1}, s_{2}$ in $(2.7)$, we get

$$
\left\|s_{1}-s_{2}\right\|_{C^{1+\beta / 2}} \leq K\left\|\psi_{1}-\psi_{2}\right\|_{C^{0, \beta / 2}}+\left\|\varphi_{1, y}-\varphi_{2, y}\right\|_{C^{0, \beta / 2}} .
$$

It then follows, upon using (2.16), (2.15), that

$$
\begin{aligned}
& \left\|s_{1}-s_{2}\right\|_{C^{1+\beta / 2}}+\left\|\varphi_{1}-\varphi_{2}\right\|_{C^{1+\beta,(1+\beta) / 2}}+\left\|\psi_{1}-\psi_{2}\right\|_{C^{1+\beta,(1+\beta) / 2}} \\
& \quad \leq K\left\{\left\|\varphi_{1}-\varphi_{2}\right\|_{C^{0}}+\left\|s_{1}-s_{2}\right\|_{C^{1}}\right\} \leq K T^{\beta / 2}\left\{\left\|\varphi_{1}-\varphi_{2}\right\|_{C^{1+\beta,(1+\beta) / 2}}+\left\|s_{1}-s_{2}\right\|_{C^{1+\beta / 2}}\right\} .
\end{aligned}
$$

Taking $T$ such that also $K T^{\beta / 2}<1$, we conclude that $s_{1} \equiv s_{2}, \varphi_{1} \equiv \varphi_{2}, \psi_{1} \equiv \psi_{2}$.

\section{Completion of the proof of Theorem 1.1.}

We first derive a priori estimates for any solution of (1.1)-(1.7), assuming that it exists in some interval $0<t<T$; these bounds will be independent of $T$.

LEMMA 3.1. The solution satisfies:

$$
\begin{array}{ll}
n_{*}<n(x, t)<2 & \text { if } \quad 0 \leq x<s(t), 0 \leq t \leq T \\
0 \leq c(x, t) \leq 1 & \text { if } \quad 0 \leq x \leq 1,0 \leq t \leq T
\end{array}
$$


and

$$
n_{x}(s(t), t)<0 \quad \text { if } \quad 0 \leq t \leq T \text {. }
$$

Proof. By (1.14)

$$
n_{t}-\left(d n_{x}\right)_{x} \geq 0 \quad \text { if } \quad n \leq n_{*} .
$$

Hence the maximum principle yields the inequality $n>n_{*}$ if $0<x<s(t), 0<t<T$. Similarly we have from (1.14) that

$$
n_{t}-\left(d n_{x}\right)_{x} \leq 0 \quad \text { if } \quad n \geq 2
$$

so that, by the maximum principle, $n<2$ if $0 \leq x \leq s(t), 0 \leq t \leq T$. The proof of (3.2) is similar. Finally, noting that, since $f\left(n_{*}, c\right)>0$,

$$
n_{t}-\left(d n_{x}\right)_{x}=f(n, c)>0
$$

near the free boundary, the (strict) inequality (3.3) follows by the maximum principle.

From (3.3) and (1.5) we deduce that

$$
\dot{s}(t)>0 \quad \text { for } \quad 0 \leq t \leq T \text {, }
$$

so that $s(t)$ is strictly monotone increasing.

Also, a standard parabolic estimate shows that $c_{x}$ is bounded independent of $T$.

LEmma 3.2. There exists a constant $M$ independent of $T$ such that

$$
\dot{s}(t) \leq M \quad \text { for } \quad 0<t<T
$$

Proof. Let

$$
Q=\{(x, t) ; \quad 0<x<s(t), \quad 0<t<T\}
$$

and introduce the operator

$$
\mathcal{L} u=u_{t}-d(u, c) u_{x x}-d_{u}(u, c) u_{x}^{2}-d_{c}(u, c) u_{x} c_{x}-f(u, c)
$$

where $c$ is the component of the solution $(s, n, c)$ of $(1.1)-(1.7)$. We shall construct a supersolution in the form

$$
\bar{n}(x, t)=\left\{\begin{array}{l}
2, \quad 0<x<s(t)-\frac{1}{M} \\
n_{*}+\left(2-n_{*}\right)\left\{2 M(s(t)-x)-M^{2}(s(t)-x)^{2}\right\}, s(t)-\frac{1}{M}<x<s(t) .
\end{array}\right.
$$

Notice that $\bar{n} \in W_{\infty}^{2,1}(Q)$ and

$$
\bar{n}_{x} \leq 0, \quad \bar{n}(s(t), t)=n_{*} .
$$

In the interval $\left[0, s(t)-\frac{1}{M}\right)$

$$
\mathcal{L} \bar{n}=-f(2, c) \geq 0 .
$$


In the interval $\left(s(t)-\frac{1}{M}, s(t)\right)$

$$
\begin{aligned}
& \bar{n}_{t}=\left(2-n_{*}\right) 2 M \dot{s}\{1-M(s-x)\} \geq 0 \quad \text { since } \quad \dot{s}(t)>0 \\
& -d(\bar{n}, c) \bar{n}_{x x}=\left(2-n_{*}\right) 2 M^{2} d(\bar{n}, c) \geq c_{0} M^{2}
\end{aligned}
$$

where $c_{0}=\left(2-n_{*}\right) 2 d_{0}>0($ see $(1.9))$,

$$
\begin{aligned}
& -d_{n}(\bar{n}, c) \bar{n}_{x}^{2} \geq 0 \quad(\text { by }(1.9)), \\
& \left|-d_{c}(\bar{n}, c) c_{x} \bar{n}_{x}\right| \leq c_{1} M,
\end{aligned}
$$

and

$$
|f(\bar{n}, c)| \leq c_{2}
$$

where $c_{1}, c_{2}$ are constants independent of $M$ and $T$. It follows that

$$
\mathcal{L} \bar{n} \geq c_{0} M^{2}-c_{1} M-c_{2}>0
$$

if $M$ is large enough. Furthermore,

$$
\bar{n}_{x}=0=n_{x} \quad \text { at } \quad x=0, \quad \bar{n}=n_{*}=n \quad \text { at } \quad x=s(t)
$$

and $\bar{n}(x, 0) \geq n_{0}(x)$ for large $M$. Hence, by comparison, $n(x, t) \leq \bar{n}(x, t)$ in $Q$ and, consequently,

$$
n_{x}(s(t), t) \geq \bar{n}_{x}(s(t), t)=-2 M\left(2-n_{*}\right)
$$

The assertion (3.5) (with another $M$ ) now follows by recalling (1.5).

From (3.5) and (3.4) we get

$$
|\dot{s}(t)| \leq M
$$

LEMMA 3.3. The solution to (1.1)-(1.7) exists and is unique, and it can be extended up to a time $T^{*}$ satisfying $\lim _{t \nearrow T^{*}} s(t)=1$.

Proof. The assertion means that if the solution exists for $0<t<T_{0}$, and if $s\left(T_{0}\right)<1$, then the solution can be continued, uniquely, to a larger interval $0<t<T_{0}+\tau(\tau>0)$. To prove this we observe that in the proof of Lemma 2.1 the size $T$ of the time interval depends on a lower bound on $\min \{s, 1-s\}$ at the initial time, and on the $L^{\infty}$ bound of the first two derivatives of the initial data. From (3.6) and $L^{p}$ estimates applied to the system with "straightened" free boundary, (2.3)-(2.9), we deduce a priori bounds on the first two derivatives of $n(x, t), c(x, t)$ at $t=T_{0}$ (in fact, even $C^{2+\alpha}$ bounds, if we use a bootstrap argument and Schauder's estimate). Since, further, $0<1-s\left(T_{0}\right)<1$, we can extend the solution, and uniquely so, to a larger interval $0<t<T_{0}+\tau$, as claimed.

We next improve the inequality (3.4):

LEMMA 3.4. There exists a positive constant $\gamma$ independent of $T$ such that

$$
\dot{s}(t) \geq \gamma \quad \text { for } \quad 0 \leq t<T .
$$


Proof. We shall construct a subsolution in the form

$$
\underline{n}(x, t)=n_{*}+\left\{\begin{array}{l}
\frac{\gamma}{2} s_{0}^{2}, \quad 0<x<s(t)-s_{0} \\
\gamma\left[s_{0}(s(t)-x)-\frac{1}{2}(x-s(t))^{2}\right], \quad s(t)-s_{0}<x<s(t) .
\end{array}\right.
$$

Notice that $\underline{n} \in W_{\infty}^{2,1}(G)$.

In the interval $\left(0, s(t)-s_{0}\right)$

$$
\mathcal{L} \underline{n}=-f\left(n_{*}+\frac{\gamma}{2} s_{0}^{2}, c\right)<0
$$

by (1.10), if $\gamma$ is sufficiently small. In the interval $\left(s(t)-s_{0}, s(t)\right)$,

$$
\begin{aligned}
& \underline{n}_{t}=\gamma\left[s_{0}-(s(t)-x)\right] \dot{s}(t) \leq \gamma M \quad \text { by }(3.6), \\
& f(\underline{n}, c) \geq f\left(n_{*}, c\right)-C_{1} \gamma
\end{aligned}
$$

and all the other terms in $\mathcal{L} \underline{n}$ are bounded by $C_{2} \gamma$, where $C_{1}, C_{2}$ are positive constants independent of $\gamma$ and $T$. Since, by (1.10), $f\left(n_{*}, c\right) \geq \mu>0$ for all $c$, we conclude that

$$
\mathcal{L} \underline{n} \leq-\mu+\gamma\left(M+C_{1}+C_{2}\right)<0
$$

if $\gamma$ is sufficiently small. We also have

$$
\underline{n}_{x}=0=n_{*} \quad \text { at } \quad x=0, \quad \underline{n}=n_{*}=n \quad \text { at } \quad x=s(t)
$$

and $\underline{n}(x, 0)<n_{0}(x)$ if $0 \leq x<s_{0}$ provided $\gamma$ is small enough; here we used the assumption $n_{0}^{\prime}\left(s_{0}\right)<0$.

By comparison we then have $\underline{n} \leq n$ in $G$ and

$$
n_{x}(s(t), t) \leq \underline{n}_{x}(s(t), t)=-\gamma s_{0},
$$

so that (3.7) holds (with another $\gamma$ ).

Combining Lemma 3.3 with 3.4 we see that there exists a finite number $T_{*}$ such that the solution exists for all $0<t<T_{*}$, and $s\left(T_{*}\right)=1$. This completes the proof of Theorem 1.1.

\section{Part II. Travelling wave solutions}

\section{Setting up the problem.}

We seek a solution to (1.1)-(1.5) in the form of a travelling wave with constant speed $c(c>0)$ :

$$
n(x, t)=u(z), \quad c(x, t)=v(z) \quad z=c t-x \in \mathbb{R}^{1} .
$$

The free boundary is given by $x=c t$, i.e., $z=0$. Then

$$
\begin{aligned}
& \left(d(u, v) u^{\prime}\right)^{\prime}-c u^{\prime}+f(u, v)=0, \quad z>0, \\
& v^{\prime \prime}-a c v^{\prime}+g(u, v)=0, \quad-\infty<z<\infty, \\
& u(z) \equiv 0, \quad z<0 .
\end{aligned}
$$

Motivated by numerical results from [2], we wish to consider only solutions such that

$$
u^{\prime}(z)>0, \quad z>0 .
$$


We impose the boundary conditions

$$
\begin{aligned}
& u(0)=u_{*} \quad\left(u_{*}=n_{*}\right) \\
& d(u(0), v(0)) u^{\prime}(0)=c u_{*}, \\
& u(\infty)=1
\end{aligned}
$$

and

$$
\begin{aligned}
& v(-\infty)=1 \\
& v(+\infty)=0
\end{aligned}
$$

As a first step we shall simplify the problem by reducing it to a system in the interval $\{z>0\}$ only.

$$
g_{0}(v)=g(0, v), \quad \beta=a c
$$

so that

$$
v^{\prime \prime}-\beta v^{\prime}+g_{0}(v)=0 \quad \text { for } \quad-\infty<z<0 .
$$

LEMma 4.1. Assume that $g_{0}(v)$ satisfies:

$$
g_{0}(1)=0, \quad \frac{d}{d v} g_{0}(v)<0 \quad \text { for all } \quad v \in \mathbb{R}^{1} .
$$

Then there exists a smooth function $\Psi(\beta, v)$ such that, for every $\beta \in(0, \infty)$, the problem

$$
v^{\prime \prime}-\beta v^{\prime}+g_{0}(v)=0 \quad \text { in } \quad(-\infty, 0), \quad v(0)=v_{0}, \quad v^{\prime}(0)=v_{1}
$$

has a bounded solution if and only if

$$
v_{1}=\Psi\left(\beta, v_{0}\right)
$$

furthermore, the bounded solution satisfies: $\lim _{z \rightarrow-\infty} v(z)=1$. The function $\Psi(\beta, v)$ has the following properties:

$$
\begin{aligned}
& \frac{d \Psi}{d v}=\beta-\frac{g_{0}(v)}{\Psi} \quad \text { in } \quad \mathbb{R}^{1} \backslash\{1\} \\
& \Psi(\beta, 1)=0
\end{aligned}
$$

and

$$
\Psi_{v}>0 \text { in } \mathbb{R}^{1}
$$

Proof. We write (4.11) as an autonomous system

$$
v^{\prime}=p, \quad p^{\prime}=\beta p-g_{0}(v) .
$$

In the $v-p$ phase plane there is only one stationary point, namely, $(1,0)$. It is a saddle point since the characteristic equation

$$
\lambda^{2}-\beta \lambda+g_{0}^{\prime}(1)=0
$$

has two real roots, one positive and one negative. Hence there are only two trajectories leaving $(1,0)$, which we shall denote by $\gamma^{+}$and $\gamma^{-}$. The velocity field of the autonomous system is 


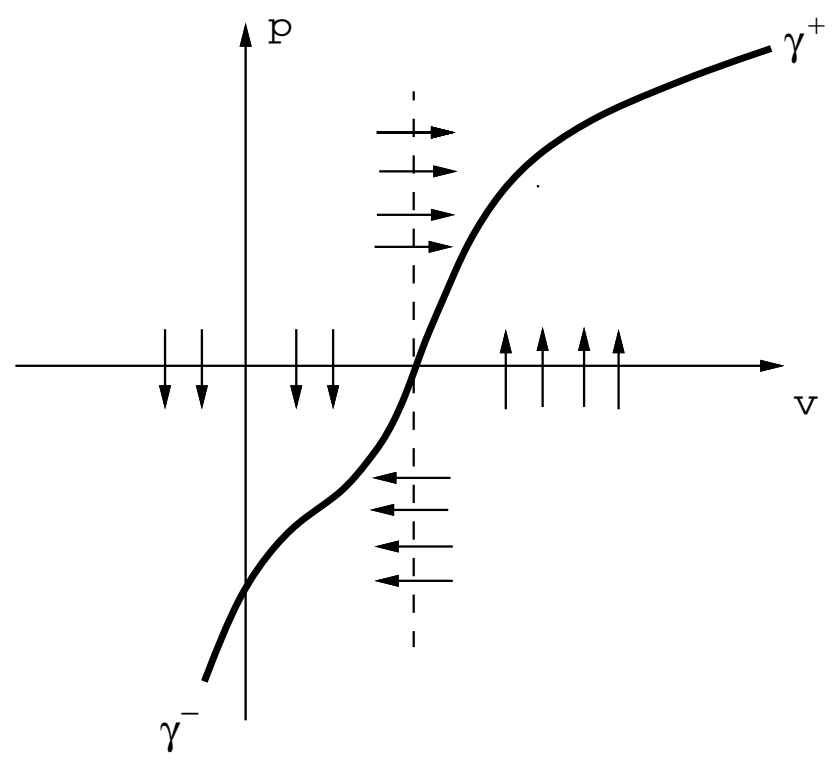

FIG. 1.

shown in Figure 1. Note that every trajectory can intersect the $v$-axis at most once. Hence $p=v^{\prime}$ can change sign at most once and, consequently, $v(-\infty)$ exists. Since there is only one stationary point, we conclude that either $|v(-\infty)|=\infty$ or $v(-\infty)=0$. Hence a solution is bounded if and only if $\left(v_{0}, v_{1}\right)$ lies on the curve $\gamma^{+} \cup\{(1,0)\} \cup \gamma^{-} \equiv \Gamma$.

Along $\gamma^{+}, p>0$ and $v>1$ so that $p^{\prime}=\beta p-g_{0}(v)>0$, whereas along $\gamma^{-}, p<0$ and $v<1$ so that $p^{\prime}<0$. It follows that $\Gamma$ can be written as a curve $p=\Psi(\beta, v)$ and, then, a solution is bounded if and only if $v_{1}=\Psi\left(\beta, v_{0}\right)$. It is clear that $\Psi(\beta, 1)=0$, and the equation in (4.14) follows from

$$
\frac{d p}{d v}=\beta-\frac{g_{0}}{p} \text {. }
$$

From the last equation we get

$$
\frac{d}{d v}\left(p \frac{d p}{d v}\right)=\beta \frac{d p}{d v}-\frac{d g_{0}}{d v}>\beta \frac{d p}{d v} .
$$

To prove (4.15) notice that the inequality holds in a neighborhood of the origin. If the inequality does not hold for all $v \neq 0$ then there is a smallest positive $v^{*}$ (or a largest negative value $v^{* *}$ ) at which $\Psi_{v}(\beta, v)$ vanishes. It follows that $\Psi_{v v} \leq 0$ at $v^{*}$ (or $\Psi_{v v} \geq 0$ at $v^{* *}$ ). But we also have $p=p\left(v^{*}\right)>0\left(p=p\left(v^{* *}\right)<0\right)$; cf. Figure 1. Therefore the left-hand side of (4.16) (with $p=\Psi(\beta, v)$ ) is negative, whereas the right-hand side vanishes at $v^{*}\left(\right.$ or $v^{* *}$ ), which is a contradiction.

REMARK 4.1. In the special case $g_{0}(v)=k(1-v)$ for some $k>0$,

$$
v=1-C e^{\lambda z}
$$

where

$$
\lambda=\frac{1}{2}\left(\beta+\sqrt{\beta^{2}+4 k}\right),
$$

and $\Psi(\beta, v)=\lambda(v-1)$.

Lemma 4.1 allows us to reformulate problem (4.1)-(4.9) as follows: 
Problem $\left(P_{0}\right)$. Find $(c, u, v)$ with $c>0$ such that

$$
\begin{aligned}
& \left(d(u, v) u^{\prime}\right)^{\prime}-c u^{\prime}+f(u, v)=0, \quad z>0, \\
& v^{\prime \prime}-a c v^{\prime}+g(u, v)=0, \quad z>0, \\
& u(0)=u_{*}, \quad d\left(u_{*}, v(0)\right) u^{\prime}(0)=c u_{*}, \quad u(\infty)=1, \\
& -v^{\prime}(0)+\Psi(a c, v(0))=0, \quad v(\infty)=0, \\
& u^{\prime}(z)>0, \quad z>0 .
\end{aligned}
$$

From (4.21) and (4.19) we see that as $z$ varies from 0 to $\infty, u=u(z)$ varies from $u_{*}$ to 1 . We can therefore try to use $u$ as an independent variable, i.e., set $z=z(u)$ as the inverse function of $u=u(z) ; u$ will vary in the interval

$$
\Omega=\left\{u_{*}<u<1\right\} .
$$

We introduce new dependent functions by

$$
Q(u)=\left.d(u, v) u^{\prime}(z)\right|_{z=z(u)}, \quad V(u)=\left.v(z)\right|_{z=z(u)} .
$$

Using the rule

$$
\frac{d}{d z}=u^{\prime} \frac{d}{d u}=\frac{Q(u)}{d(u, v)} \frac{d}{d u}
$$

we can transform problem $\left(P_{0}\right)$ into the following problem:

Problem $\left(P_{1}\right)$. Find $(c, Q, V)$ with $c>0$ such that

$$
\begin{aligned}
& Q Q_{u}-c Q+d(u, V) f(u, V)=0 \quad \text { in } \Omega, \\
& \frac{Q}{d(u, V)}\left(\frac{Q}{d(u, V)} V_{u}\right)_{u}-a c \frac{Q}{d(u, V)} V_{u}+g(u, V)=0 \text { in } \Omega, \\
& Q\left(u_{*}\right)=c u_{*}, \quad Q(1)=0, \\
& -\frac{Q\left(u_{*}\right)}{d\left(u_{*}, V\left(u_{*}\right)\right)} V_{u}+\Psi\left(a c, V\left(u_{*}\right)\right)=0, \quad V(1)=0, \\
& Q>0 \text { in } \Omega, \\
& \int_{u_{*}}^{1} \frac{d(s, V(s))}{Q(s)} d s=\infty .
\end{aligned}
$$

Indeed we have:

Lemma 4.2. Problems $\left(P_{0}\right)$ and $\left(P_{1}\right)$ are equivalent.

Proof. Given a solution to problem $\left(P_{0}\right)$, define $z=z(u)$ as the inverse of $u=u(z)$ and define $Q, V$ by (4.22). Then (4.23), (4.24), (4.26), (4.27) and the first boundary condition in (4.25) hold, and it remains to prove that $Q(1)=0$. Since

$$
z=\int_{u_{*}}^{u} \frac{d(s, V(s))}{Q(s)} d s
$$

and $u(\infty)=1$, we also have (4.28). Finally, note that the existence of $u(\infty)$ and $v(\infty)$ implies by standard ODE theory that $u^{\prime \prime}, v^{\prime \prime}$ are bounded, and then, by a simple argument in real analysis, $u^{\prime}(\infty), v^{\prime}(\infty)$ must exist and equal to zero. Taking $u \rightarrow 1$ in the first relation of (4.22) we then deduce that $Q(1)=0$. 
Conversely consider a solution to problem $\left(P_{1}\right)$ and define $u=u(z)$ by

$$
u^{\prime}(z)=\frac{Q(u)}{d(u, V(u))}, \quad u(0)=u_{*}
$$

and $v(z)=V(u(z))$. Then (4.29) holds, and one can easily show that all the equations in problem $\left(P_{0}\right)$ are satisfied.

\section{Statement of the main result.}

In the sequel we shall work primarily (but not exclusively) with problem $\left(P_{1}\right)$. We shall need some assumptions that include all those made in (1.9)-(1.11) (except for the condition $f(2, c) \leq 0$, which will not be needed). Using the variables $(u, v)$ instead $(n, c)$, we introduce the set (which coincides with $G$ )

$$
S=\left\{(u, v) ; \quad u_{*} \leq u \leq 1, \quad 0 \leq v \leq 1\right\}
$$

We assume:

the function $d(u, v)$ is in $C^{1}(S)$, and

$$
0<d_{0} \leq d(u, v) \leq d_{1}<\infty, \quad d_{u} \leq 0, \quad d_{v} \geq 0, \quad a d \leq 1
$$

where $a$ is the constant appearing in (4.2) and (4.18);

$$
\begin{aligned}
& \text { the function } f(u, v) \text { is in } C^{1}(S) \text { and } \\
& f(1,0)=0, \quad f_{u}(1,0)<0, \quad f(u, 0)>0 \text { for } u_{*} \leq u<1, \\
& f_{v}(u, v)>0 \text { in } S ;
\end{aligned}
$$

the function $g(u, v)$ is in $C^{1}(S)$ and

$$
\begin{aligned}
& g(0,1)=0, \quad g(u, 0) \geq 0 \quad \text { and } \quad g(u, 1) \leq 0 \quad \text { for } \quad u_{*} \leq u \leq 1 \\
& g_{u}(u, v)<0, \quad g_{v}(u, v)<0 \quad \text { in } S ;
\end{aligned}
$$

there exists a positive number $\ell$ such that $\ell\left(1-u_{*}\right)>1$ and

$$
\max _{0 \leq v \leq \ell(1-u)} \frac{f(u, v)}{d(u, v)}+\frac{1}{\ell} g(u, \ell(1-u)) \leq 0 \quad \text { for } \quad 1-\frac{1}{\ell} \leq u \leq 1 .
$$

Note that $1-\frac{1}{\ell}>u_{*}$.

THEOREM 5.1. If the conditions (5.1)-(5.4) hold then problem $\left(P_{0}\right)$ has at least one solution $(c, u, v)$ and $v^{\prime}(z)<0$ for $0<z<\infty$.

The proof is given in the next three sections. In Section 6 we solve, for a given $V=V(u)$, the equation (4.23) for $(c, Q)$ under the conditions $Q\left(u_{*}\right)=c u_{*}, Q(1)=0$. Substituting this solution into equation (4.24), we solve the resulting equation, in Section 7, and denote the solution by $W$; this defines a mapping $T: V \rightarrow W$. For technical reasons we shall actually consider the $V$-equation only in the interval $u_{*} \leq u<1-\varepsilon(\varepsilon>0)$, in order to avoid the degeneracy of the leading coefficient at $u=1$. Thus the mapping $T$ depends on $\varepsilon$ (and will be denoted by $T_{\varepsilon}$ ), and we shall prove that it has at least one fixed point. In Section 8 we let $\varepsilon \rightarrow 0$ and, invoking the equivalence established in Lemma 4.2 , prove that the solution $\left(c_{\varepsilon}, Q_{\varepsilon}, V_{\varepsilon}\right)$ of the fixed point of $T_{\varepsilon}$ converges to a solution of problem $\left(P_{1}\right)$. 
6. Solution for $(\boldsymbol{c}, Q)$, given $\boldsymbol{V}(\boldsymbol{u})$. For any small $\varepsilon>0$ we introduce the space

$$
X_{\varepsilon}=\left\{V \in C^{0}\left[u_{*}, 1\right], \quad 0 \leq V \leq 1, \quad V \equiv 0 \quad \text { on } \quad[1-\varepsilon, 1]\right\} .
$$

Given $V(u)$ in $X_{\varepsilon}$, consider the problem:

Problem $\left(P_{2}\right)$. Find $(c, Q)$ with $c>0$ such that

$$
\begin{aligned}
& Q Q_{u}-c Q+d(u, V(u)) f(u, V(u))=0 \quad \text { in } \Omega, \\
& Q\left(u_{*}\right)=c u_{*}, \quad Q(1)=0, \quad Q>0 \quad \text { in } \Omega .
\end{aligned}
$$

THEOREM 6.1. Under the assumptions (5.1), (5.2) there exists a unique solution to (6.1), (6.2); furthermore, the following estimates hold:

$$
\begin{gathered}
c_{1} \leq c \leq c_{2} \text { where } \\
c_{1}=\sqrt{d_{0}} \max _{\bar{\Omega}}\left\{\frac{1}{u} \int_{u_{*}}^{u} \frac{f(s, 0)}{s} d s\right\}^{1 / 2}, \quad c_{2}=2 \sqrt{d_{1}} \max _{\bar{\Omega}}\left\{\frac{1}{u} \int_{u_{*}}^{u} \frac{f(s, 1)}{s} d s\right\}^{1 / 2}, \\
c_{3}(1-u) \leq Q(u) \leq c_{2} u \quad \text { in } \Omega \text { where } \\
c_{3}=\frac{1}{2}\left\{-c_{2}+\left[c_{2}^{2}+4 d_{0} \min _{\bar{\Omega}} \frac{f(s, 0)}{1-s}\right]^{1 / 2}\right\},
\end{gathered}
$$

and

$$
Q(u) \leq \sqrt{2 d_{1}}\left[\int_{u}^{1} f(s, V(s)) d s\right]^{1 / 2} \leq \sqrt{2 d_{1}}\|f\|_{L^{\infty}} \sqrt{1-u} .
$$

Note, by (5.2), that $0<c_{1}<c_{2}$ and $c_{3}>0$.

To prove the theorem we consider first the more general Problem $\left(P_{3}\right)$. Find $(c, Q)$ with $c>0$ such that

$$
\begin{aligned}
& Q Q_{u}-c Q+F(u)=0 \quad \text { in } \Omega=\left(u_{*}, 1\right), \\
& Q\left(u_{*}\right)=c u_{*}, \quad Q(1)=0, \quad Q(u)>0 \quad \text { in } \Omega
\end{aligned}
$$

where

$$
F \in C^{0}\left[u_{*}, 1\right], \quad F(1)=0, \quad F(u)>0 \quad \text { in }\left(u_{*}, 1\right) .
$$

Lemma 6.2. Problem $\left(P_{3}\right)$ admits a unique solution $(c, Q)$. The solution has the following properties:

$$
\begin{aligned}
& c_{F}<c<2 c_{F} \quad \text { where } \quad c_{F}=\max _{\bar{\Omega}}\left\{\frac{1}{u} \int_{u_{*}}^{u} \frac{F(s)}{s} d s\right\}^{1 / 2}, \\
& 0<Q(u)<c u \quad \text { in } \Omega, \\
& \lambda(u)(1-u) \leq Q(u) \leq \Lambda(u)(1-u) \text { in } \Omega
\end{aligned}
$$

where

$$
\begin{aligned}
& \lambda(u)=\frac{1}{2}\left\{-c+\left[c^{2}+4 \min _{[u, 1]} \frac{F(s)}{1-s}\right]^{1 / 2}\right\} \in(0, \infty), \\
& \Lambda(u)=\frac{1}{2}\left\{-c+\left[c^{2}+4 \max _{[u, 1]} \frac{F(s)}{1-s}\right]^{1 / 2}\right\} \in(0, \infty) \cup\{\infty\},
\end{aligned}
$$




$$
Q(u)<\left\{2 \int_{u}^{1} F(s) d s\right\}^{1 / 2} \leq\left\{2|F|_{L^{\infty}(\Omega)}\right\}^{1 / 2}(1-u)^{1 / 2} \text { in } \Omega
$$

and

$$
c=\int_{u_{*}}^{1} \frac{F(s)}{Q(s)} d s>0
$$

Note that if $F_{u}(1,0)=-\infty$ then $\Lambda(u)=\infty$.

Proof. We first prove uniqueness. Suppose $\left(c_{1}, Q_{1}\right)$ and $\left(c_{2}, Q_{2}\right)$ are two solutions. It suffices to show that $c_{1}=c_{2}$. We proceed by contradiction, assuming that $c_{1}>c_{2}$. Then $Q_{1}\left(u_{*}\right)>Q_{2}\left(u_{*}\right)$, and we introduce

$$
\bar{u}=\sup \left\{u \in\left[u_{*}, 1\right], \quad Q_{1}>Q_{2} \quad \text { in }\left[u_{*}, u\right]\right\}
$$

If $\bar{u}<1$ then $Q_{1}(\bar{u})=Q_{2}(\bar{u})$ and $\frac{d}{d u}\left(Q_{1}-Q_{2}\right) \leq 0$ at $\bar{u}$. However from (6.6) we get

$$
\left.\frac{d}{d u}\left(Q_{1}-Q_{2}\right)\right|_{u=\bar{u}}=c_{1}-c_{2}-F(\bar{u})\left[\frac{1}{Q_{1}(\bar{u})}-\frac{1}{Q_{2}(\bar{u})}\right]=c_{1}-c_{2}>0
$$

a contradiction. We conclude that $\bar{u}=1$, i.e., $Q_{1}>Q_{2}$ in $\left[u_{*}, 1\right)$. Integrating $(6.6)$ over $\left[u_{*}, 1\right]$ for $\left(c_{1}, Q_{1}\right)$ and $\left(c_{2}, Q_{2}\right)$ and taking the difference, we get, after using (6.7),

$$
\begin{aligned}
0>\frac{1}{2}\left(c_{2}^{2}-c_{1}^{2}\right) u_{*}^{2} & =\left[\frac{1}{2} Q_{1}^{2}-\frac{1}{2} Q_{2}^{2}\right]_{u=u_{*}}^{u=1}=\int_{u_{*}}^{1}\left(Q_{1} \frac{\partial Q_{1}}{\partial u}-Q_{2} \frac{\partial Q_{2}}{\partial u}\right) d u \\
& =\int_{u_{*}}^{1}\left(c_{1} Q_{1}-c_{2} Q_{2}\right) d u>0
\end{aligned}
$$

a contradiction.

To prove existence we extend $F(u)$ by 0 to $u>1$. Denote by $Q(c, u)(c>0)$ the solution to

$$
Q_{u}=c-\frac{F}{Q}, \quad Q\left(u_{*}\right)=c u_{*}
$$

and denote by $\left[u_{*}, \gamma(c)\right)$ the maximal existence interval where $Q>0$. The set

$$
\mathcal{A}=\{c>0 ; \quad \gamma(c)>1\}
$$

is clearly an open set. We claim that

$$
\left[0, c_{F}\right) \cap \mathcal{A}=\varnothing
$$

Indeed, since $F>0$ in $\Omega,(6.14)$ gives $Q_{u}<c$ so that, by integration, $Q<c u$ and, consequently,

$$
Q_{u}<c-\frac{F}{c u}
$$

Again, by integration,

$$
Q<c u-\int_{u_{*}}^{u} \frac{F(s)}{c s} d s=\frac{u}{c}\left\{c^{2}-\frac{1}{u} \int_{u_{*}}^{u} \frac{F(s)}{s} d s\right\} .
$$


It follows that if $c<c_{F}$ then $\gamma(c)<1$, i.e., (6.15) holds.

We next prove that

$$
\left[2 c_{F}, \infty\right) \subset \mathcal{A}
$$

Indeed, denote by $\gamma_{1}(c)$ the smallest value of $u$ in $\left[u_{*}, \gamma(c)\right]$ such that $Q=\frac{1}{2} c u$, i.e.,

$$
\gamma_{1}(c)=\sup \left\{s \leq \gamma(c), \quad Q(u)>\frac{1}{2} c u \quad \text { in }\left[u_{*}, s\right]\right\}
$$

If $\gamma_{1}=\gamma_{1}(c) \leq 1$ then, integrating (6.14) over $\left[u_{*}, \gamma_{1}(c)\right]$, we obtain

$$
\begin{aligned}
Q\left(\gamma_{1}(c)\right) & =c \gamma_{1}-\int_{u_{*}}^{\gamma_{1}} \frac{F}{Q}>c \gamma_{1}-\int_{u_{*}}^{\gamma_{1}} \frac{2 F(s)}{c s} d s \\
& =\frac{c \gamma_{1}}{2}\left\{2-\frac{4}{c^{2}} \frac{1}{\gamma_{1}} \int_{u_{*}}^{\gamma_{1}} \frac{F(s)}{s} d s\right\} \geq \frac{c \gamma_{1}}{2} \quad \text { if } \quad c \geq 2 c_{F}
\end{aligned}
$$

which is a contradiction. Hence $\gamma(c) \geq \gamma_{1}(c)>1$ if $c \geq 2 c_{F}$ and (6.16) is proved.

Now define $c_{0}=\inf \{c ; c \in \mathcal{A}\}$. By continuity, $\gamma\left(c_{0}\right) \geq 1$ and, from (6.16) and the proof of (6.15), $c_{F}<c_{0}<2 c_{F}$. If $Q\left(c_{0}, 1\right)>0$ then, as $\mathcal{A}$ is open, there are values of $c$ smaller than $c_{0}$ (and near $c_{0}$ ) for which $Q(c, u)>0$ if $u_{*} \leq u \leq 1$, which contradicts the definition of $c_{0}$. We conclude that $Q\left(c_{0}, 1\right)=0$ and thus $\left(c_{0}, Q\left(c_{0}, u\right)\right)$ is a solution to problem $\left(P_{3}\right)$. For simplicity we shall denote this solution by $(c, Q)$.

Integrating $(6.6)$ over $[u, 1]$ we have

$$
\frac{1}{2}\left[Q^{2}\right]_{u}^{1}>-\int_{u}^{1} F(s) d s
$$

so that

$$
Q^{2}(u)<2 \int_{u}^{1} F(s) d s
$$

and (6.12) follows. Integrating (6.14) over $\left[u_{*}, 1\right]$ and using (6.7), the relation (6.13) also follows.

It remains to prove (6.11). We first derive the upper bound on $Q$. If we define

$$
\delta=\inf \left\{\tilde{s}>0 ; \quad Q(u)<\tilde{s}+\Lambda(u)(1-u) \text { in }\left[u_{*}, 1\right]\right\}
$$

then it suffices to show that $\delta=0$. Without loss of generality here we may assume that $\Lambda(1-)<\infty$. Then $\Lambda(u)$ is continuous and, if $\delta>0$, there exists a point $u_{1} \in\left[u_{*}, 1\right)$ such that $Q\left(u_{1}\right)=\delta+\Lambda\left(u_{1}\right)\left(1-u_{1}\right)$ and

$$
\left.\frac{d Q}{d u}\right|_{u=u_{1}} \leq\left.\frac{d}{d u}[\Lambda(u)(1-u)]\right|_{u=u_{1}} \leq-\Lambda\left(u_{1}\right)
$$

since $\Lambda(u)$ is monotone decreasing; the first inequality is actually an equality if $u^{*}<u_{1}<1$. Using (6.14) we get

$$
-\Lambda\left(u_{1}\right) \geq c-\frac{F\left(u_{1}\right)}{\delta+\Lambda\left(u_{1}\right)\left(1-u_{1}\right)} \geq c-\frac{F\left(u_{1}\right)}{\Lambda\left(u_{1}\right)\left(1-u_{1}\right)}
$$

that is

$$
-\Lambda^{2}\left(u_{1}\right)>c \Lambda\left(u_{1}\right)-\frac{F\left(u_{1}\right)}{1-u_{1}} \geq c \Lambda\left(u_{1}\right)-\max _{\left[u_{1}, 1\right]} \frac{F(s)}{1-s}
$$


However, by definition of $\Lambda(u)$,

$$
\Lambda^{2}(u)+c \Lambda(u)-\max _{[u, 1]} \frac{F(s)}{1-s}=0
$$

which is a contradiction.

Similarly we define

$$
\delta=\inf \{s>0 ; \quad Q(u)>\lambda(u)(1-u)-s\}
$$

and show that $\delta=0$.

Proof of Theorem 6.1. Applying Lemma 6.2 with $F(u)=d(u, V(u)) f(u, V(u))$ and using (5.1), (5.2) and the fact that $V(u)$ is in $X_{\varepsilon}$, the assertions of Theorem 6.1 immediately follow. (The assumptions $d_{u} \leq 0, d_{v} \geq 0, a d \leq 1$ are not needed for Theorem 6.1).

\section{A fixed point $\left(c_{\varepsilon}, Q_{\varepsilon}, V_{\varepsilon}\right)$.}

Given $V \in X_{\varepsilon}$, let $(c, Q)$ denote the solution of (6.1), (6.2) and consider the problem: Problem $\left(P_{4}\right)$. Find $W(u)$ such that

$$
\begin{aligned}
& \frac{Q}{d}\left(\frac{Q}{d} W_{u}\right)_{u}-a c \frac{Q}{d} W_{u}+g(u, W)=0 \text { in } \Omega_{\varepsilon}=\left[u_{*}, 1-\varepsilon\right], \\
& -\frac{Q}{d} W_{u}+\Psi(a c, W)=0 \quad \text { at } u=u_{*}, \\
& W(u)=0 \quad \text { in }[1-\varepsilon, 1]
\end{aligned}
$$

where $d=d(u, V(u))$.

Lemma 7.1. Under the assumptions (5.1)-(5.3) there exists a unique solution $W$ to problem $\left(P_{4}\right)$, and the following inequalities hold:

$$
\begin{aligned}
& 0 \leq W \leq 1 \quad \text { in } \quad \Omega_{\varepsilon} \\
& -M_{\varepsilon} \leq W_{u} \leq 0 \quad \text { in } \quad \Omega_{\varepsilon}, \\
& \left|W_{u}\left(u_{*}\right)\right| \leq c_{4}
\end{aligned}
$$

where $M_{\varepsilon}$ is a constant which depends on $\varepsilon$ but not on $V \in X_{\varepsilon}$, and $c_{4}$ is independent of $\varepsilon$ and $V$.

Proof. Extend the definition of $g(u, w)$ to all $w \in \mathbb{R}^{1}$ such that $g_{w}<0$. By Theorem 6.1

$$
c_{3} \varepsilon \leq Q<c_{2} \text { in }\left[u_{*}, 1-\varepsilon\right],
$$

so that the differential equation (7.1) is non-degenerate in $\Omega_{\varepsilon}$. The inequalities $g_{w}<0$ and $\Psi_{w}>0$ will enable us to use the comparison principle for the system (7.1)-(7.3). For any function $\tilde{W}(u)$ satisfying $0 \leq \tilde{W}(u) \leq 1$, we set $g=g(u, \tilde{W}(u)), \Psi=\Psi(a c, \tilde{W}(u))$ and denote the corresponding solution of (7.1)-(7.3) by $W(u)$. Since

$$
\begin{aligned}
& g(u, 0) \geq g(u, \tilde{W}(u)) \geq g(u, 1), \\
& \Psi(a c, 0) \leq \Psi(a c, \tilde{W}(u)) \leq \Psi(a c, 1),
\end{aligned}
$$

the comparison principle shows that $0 \leq W(u) \leq 1$. We can therefore apply the Schauder fixed point argument to the mapping $\tilde{W} \rightarrow W$ to deduce the existence of a solution to (7.1)-(7.3). Uniqueness of the solution of (7.1)-(7.3) follows again by a comparison argument. 
Next, differentiating (7.1) and setting $Z=\frac{Q}{d} W_{u}$, we get

$$
\left(\frac{Q}{d} Z_{u}\right)_{u}-a c Z_{u}+g_{v} \frac{d}{Q} Z+g_{u}=0
$$

Also

$$
\left.Z\right|_{u=u_{*}}=\Psi\left(a c, W\left(u_{*}\right)\right)<0,\left.\quad Z\right|_{u=1-\varepsilon} \leq 0 .
$$

Since $g_{v}<0$ and $g_{u}<0$, the maximum principle yields $Z \leq 0$, i.e., $W_{u} \leq 0$. Finally, (7.6) and the first inequality in (7.5) follow from the fact that the equation (7.1) is non-degenerate (i.e., from (7.7)).

We shall now combine Lemma 7.1 with Theorem 6.1 . For every $V \in X_{\varepsilon}$ we define $(c, Q)$ by Theorem 6.1 and $W$ by Lemma 7.1 , and introduce the mapping $T_{\varepsilon}$ by

$$
T_{\varepsilon} V=W
$$

Clearly $T_{\varepsilon}$ maps $X_{\varepsilon}$ into itself, and its image lies in a compact subset of $X_{\varepsilon}$ (since $\left|W_{u}\right| \leq$ $M_{\varepsilon}$ ). By the uniqueness parts of Theorem 6.1 and Lemma 7.1 it also easily follows that $T_{\varepsilon}$ is continuous. Invoking the Schauder fixed point theorem, we conclude that there exists at least one fixed point for $T_{\varepsilon}$. We shall denote it by $\left(c_{\varepsilon}, Q_{\varepsilon}, V_{\varepsilon}\right)$.

We define a function $u_{\varepsilon}(z)$ by

$$
z=\int_{u_{*}}^{u_{\varepsilon}(z)} \frac{d\left(s, V_{\varepsilon}(s)\right)}{Q_{\varepsilon}(s)} d s, \quad z \in\left[0, Z_{\varepsilon}\right]
$$

where

$$
Z_{\varepsilon}=\int_{u_{*}}^{1-\varepsilon} \frac{d\left(s, V_{\varepsilon}(s)\right)}{Q_{\varepsilon}(s)} d s
$$

and introduce also the function

$$
v_{\varepsilon}(z)=V_{\varepsilon}\left(u_{\varepsilon}(z)\right) \text {. }
$$

Then $\left(c_{\varepsilon}, u_{\varepsilon}(z), v_{\varepsilon}(z)\right)$ form a solution of the system $(4.17)-(4.21)$ in $\left[0, Z_{\varepsilon}\right]$, but without the conditions $u(\infty)=1, v(\infty)=0$.

By (6.9)-(6.11), the functions $Q_{\varepsilon}(u)$ are uniformly bounded from above and below by two positive constants, for $u_{*} \leq u \leq 1-\delta$ (for any $\delta>0$ ), and the same is true for the constants $c_{\varepsilon}$. Using also the estimates of Lemma 7.1 , we deduce that $u_{\varepsilon}(0), u_{\varepsilon}^{\prime}(0), v_{\varepsilon}(0), v_{\varepsilon}^{\prime}(0)$, are uniformly bounded. Hence, we can choose a subsequence $\varepsilon \downarrow 0$ such that

$$
\left(c_{\varepsilon}, u_{\varepsilon}, v_{\varepsilon}\right) \rightarrow(c, u, v)
$$

where $(c, u, v)$ is a solution of $(4.17)-(4.21)$ for $0<z<z_{0}$, where

$$
z_{0}=\lim _{\varepsilon \downarrow 0} Z_{\varepsilon}
$$

The corresponding limits $(Q, V)$ of $\left(Q_{\varepsilon}, V_{\varepsilon}\right)$ also exist, and $Q(u)>0$ if $u_{*}<u<1, Q(1)=0$.

LEMMA 7.2. If $z_{0}=\infty$ then $(c, u, v)$ is a solution to problem $\left(P_{0}\right)$.

Proof. The only assertions that still need to be proved are:

$$
u(\infty)=1, \quad v(\infty)=0
$$


We know that $u(\infty), v(\infty)$ exist. Consequently (by ODE theory) $u^{\prime \prime}$ and $v^{\prime \prime}$ are bounded and then $u^{\prime}(\infty), v^{\prime}(\infty)$ must exist and be equal to zero. The relation (4.30) holds for $0<z<\infty$, and taking $z \rightarrow \infty$ we get $Q(u(\infty))=0$, so that $u(\infty)=1$. Finally, from (4.17) we deduce that

$$
d(1, v(\infty)) u^{\prime \prime}+f(1, v(\infty)) \rightarrow 0 \quad \text { if } \quad z \rightarrow \infty .
$$

From this it follows that $f(1, v(\infty))=0$ (otherwise $u^{\prime}(z)$ will not converge to zero as $z \rightarrow \infty$ ), so that, by $(5.2), v(\infty)=0$.

\section{Proof of Theorem 5.1.}

In Section 7 we established the existence of a fixed point $\left(c_{\varepsilon}, u_{\varepsilon}, v_{\varepsilon}\right)$, or $\left(c_{\varepsilon}, Q_{\varepsilon}, V_{\varepsilon}\right)$, for the mapping $T_{\varepsilon}: X_{\varepsilon} \rightarrow X_{\varepsilon}$, and also proved that $\partial V_{\varepsilon} / \partial u \leq 0$. In this section we shall replace $X_{\varepsilon}$ by the subset

$$
\begin{aligned}
Y_{\varepsilon}=\left\{V \in C^{0}\left[u_{*}, 1\right] ; \quad 0 \leq V \leq 1, \quad V_{u} \leq 0,\right. \\
\left.V \equiv 0 \quad \text { on }[1-\varepsilon, 1], \quad \text { and } \quad V(u) \leq \ell(1-u) \text { in }\left[1-\frac{1}{\ell}, 1\right]\right\}
\end{aligned}
$$

where $\ell$ is the positive number appearing in the condition (5.4).

LEMma 8.1. Under the assumptions (5.1)-(5.4), $T_{\varepsilon}$ has a fixed point in $Y_{\varepsilon}$.

Proof. We only need to show that $T_{\varepsilon}$ maps $Y_{\varepsilon}$ into itself; the rest of the analysis is as before. If we set $T_{\varepsilon} V=W$ then, as before, $W_{u} \leq 0$, and so all we need to show is that

$$
W(u) \leq \ell(1-u) \quad \text { in }\left[1-\frac{1}{\ell}, 1-\varepsilon\right] .
$$

Consider the function $\bar{W}(u)=\ell(1-u)$ in $\left[1-\frac{1}{\ell}, 1-\varepsilon\right]$. It satisfies:

$$
\bar{W}\left(1-\frac{1}{\ell}\right)=1 \geq W\left(1-\frac{1}{\ell}\right), \quad \bar{W}(1-\varepsilon)=\ell \varepsilon>0=W(1-\varepsilon) .
$$

Also

$$
\begin{aligned}
\mathcal{L} W & \equiv \frac{Q}{d}\left(\frac{Q}{d} \bar{W}_{u}\right)_{u}-\alpha c \frac{Q}{d} \bar{W}_{u}+g(u, \bar{W}) \\
& =-\ell\left\{\frac{Q}{d} \frac{Q_{u}}{d}-\frac{Q^{2}}{d} \frac{d_{u}+d_{v} V_{u}}{d^{2}}\right\}+\ell a c \frac{Q}{d}+g(u, \bar{W}) \\
& =-\frac{\ell}{d^{2}}\{(c-a c d) Q-d f\}+\frac{Q^{2} \ell}{d^{3}}\left(d_{u}+d_{v} V_{u}\right)+g(u, \bar{W}) \text { by (6.1). }
\end{aligned}
$$

Since $d_{u} \leq 0, d_{v} \geq 0, V_{u} \leq 0,1-a d \geq 0, Q>0$, we get

$$
\mathcal{L} W \leq \ell\left\{\frac{f(u, V(u))}{d(u, V(u))}+\frac{1}{\ell} g(u, \ell(1-u))\right\} \leq 0 \quad \text { in } \quad\left[1-\frac{1}{\ell}, 1-\varepsilon\right],
$$

by (5.4); here we used the inequality $V(u) \leq \ell(1-u)$ in $\left[1-\frac{1}{\ell}, 1\right]$. Thus $\bar{W}$ is a supersolution, and (8.2) follows by comparison.

We shall henceforth work with the solution $\left(c_{\varepsilon}, u_{\varepsilon}, v_{\varepsilon}\right)$, or $\left(c_{\varepsilon}, Q_{\varepsilon}, V_{\varepsilon}\right)$, corresponding to the space $Y_{\varepsilon}$ and with the corresponding limits $(c, u, v)$ (see (7.10)) and $(c, Q, V)$. We shall prove 
that $(c, u, v)$ is a solution to problem $\left(P_{0}\right)$. In view of Lemma 7.2 , all we need to prove is the following:

LEMMA 8.2. Under the assumptions (5.1)-(5.4) the following relations hold:

$$
z_{0}=\int_{u_{*}}^{1} \frac{d(s, V(s))}{Q(s)} d s=\infty .
$$

Proof. Recalling that $f(1,0)=0$ and using Lemma 8.1 we have

$$
f\left(u, V_{\varepsilon}(u)\right) \leq\left\|f_{u}\right\|_{L^{\infty}}(1-u)+\left\|f_{v}\right\|_{L^{\infty}} V_{\varepsilon}(u) \leq M(1-u)
$$

for $u \in\left[1-\frac{1}{\ell}, 1-\varepsilon\right]$. Using this in (6.5) we get $Q_{\varepsilon}(u) \leq M(1-u)$ with another constant $M$, independent of $\varepsilon$. It follows that

$$
Q(u) \leq M(1-u) \quad \text { in } \quad\left[1-\frac{1}{\ell}, 1\right]
$$

and, since $d(u, V(u)) \geq d_{0}>0$, the assertion (8.3) follows.

\section{Application to corneal epthelial wound healing.}

In the model that appears in [2] (see also [1]), after non-dimensionalization,

$$
\begin{aligned}
& \frac{\partial N}{\partial t}=\frac{\partial}{\partial X}\left[(\alpha+0.1 \alpha C) \frac{\partial N}{\partial X}\right]+\frac{\partial}{\partial X}(e(X, t) N)+F(N, C), \\
& \frac{\partial C}{\partial t}=D_{C} \frac{\partial^{2} C}{\partial X^{2}}+G(N, C)
\end{aligned}
$$

where

$$
\begin{aligned}
& F(N, C)=+(0.9+0.1 C)\left(2 N-N^{2}\right)-N, \\
& G(N, C)=A+B(N)-\mu N h(C)-\delta C, \\
& h(C)=\frac{C}{\hat{C}+C}, \quad \delta=242, \\
& B(N)=B(0) \chi_{\{N=0\}} \quad B(0)>0, \quad \alpha=0.012, \quad D_{C}=6, \quad \mu=2 \times 10^{4} .
\end{aligned}
$$

Here $e(X, t) N$ accounts for the physiological electric field and it thus depends on the proximity of $X$ to the free boundary. It seems, however, more natural to replace $e(X, t) N$ by a function $k(N) N_{X}$ where $k\left(n_{*}\right)>0, k^{\prime}(N) \leq 0$ if $N>n_{*}$, and $k(N)=0$ if $N>n_{*}+\varepsilon_{0}$ for some $\varepsilon_{0}>0$. We shall take

$$
k(N)=e_{0} E(N), \quad e_{0}>0
$$

so that $E^{\prime}(N) \leq 0$; it will be convenient to set $e_{0}=\alpha e, e>0$.

Let $X=\sqrt{\alpha} x, N=n(x, t), C=1+C^{*} c(x, t)$ where, by [2], $C^{*} \sim 5-50$. Writing the system (9.1), (9.2) (with $e(X, t) N$ replaced by $\alpha e E(N))$ in the form (1.1), (1.2), we then have

$$
d(n, c)=1.1+b c+e E(n)
$$

where $a=\alpha / D_{C}=2 \times 10^{-3}, b=0.1 C^{*}$,

$$
f(n, c)=(1+b c) n(1-n)+b n c
$$


and

$$
g(n, c)=\frac{a}{C^{*}} G\left(n, 1+C^{*} c\right) .
$$

It is easily seen that all the assumptions made in Theorem 1.1 are satisfied.

In order to verify the assumptions of Theorem 5.1, we observe that the numerical results obtained in $[1,2]$, which exhibit a travelling wave solution, imply that

$$
G(1,1)=0 \quad G\left(0,1+C^{*}\right)=0,
$$

so that

$$
A=\delta+\mu h(1), \quad C^{*}=\frac{1}{\delta}(\mu h(1)+B(0))
$$

Setting

$$
\theta=\frac{\mu h(1)}{\mu h(1)+B(0)}, \quad 0<\theta<1
$$

we get

$$
g(n, c)=a \delta\left\{-c+\theta(1-n)+\theta n\left(1-\frac{h\left(1+C^{*} c\right)}{h(1)}\right)+(1-\theta) \chi_{\{n=0\}}\right\} .
$$

We easily check that the functions $d, f, g$ then satisfy all the assumptions in (5.1)-(5.3). Thus it remains to show that condition (5.4) also holds; this is more dedicate. We note that whereas in [1] (and also in [2]) $\hat{C}$ is taken as 3, so that $h(1)=\frac{1}{4}$, the numerical graph in Figure 2 in [1] shows that actually $1+C^{*}=5$ in case $B(0)=0$, so that, from the second relation in (9.5),

$$
h(1)=\frac{\delta C^{*}}{\mu} \sim \frac{1}{20} \text {. }
$$

We shall take an intermediate value, $h(1)=\frac{1}{10}$, so that $\hat{C}=9$. Then

$$
g(u, v)=0.4\left\{-v+\theta(1-u)-\theta C^{*} \frac{u v}{1+b v}\right\}
$$

in the healed region. Clearly

$$
\max _{0 \leq v \leq w} \frac{f(u, v)}{d(u, v)} \leq u(1-u)+\frac{b u w}{1+b w}
$$

and, with $v=\ell(1-u)$,

$$
\left.\frac{g(u, v)}{\ell}\right|_{v=\ell(1-u)} \leq-4 \theta b \frac{u(1-u)}{1+b w} \quad \text { if } \quad \ell>1 \geq \theta .
$$

Hence the condition (5.4) holds if

$$
1+\frac{\ell b}{1+b w}-\frac{4 \theta b}{1+b w} \leq 0 \quad \text { for } \quad 0 \leq w \leq 1 .
$$

This inequality is satisfied for some $\ell, \theta$ near 1 provided $2 b>1$, which is indeed the case since $b=0.1 C^{*}$ and $C^{*}>8$ by $(9.5)$.

We conclude that for the relevant range of parameters that occur in $[1,2]$ the functions $d, f, g$ satisfy all the assumptions made in Theorem 1.1 and all the assumptions (5.1)-(5.3) made in Theorem 5.1. As for the last assumption (5.4) made in Theorem 5.1, it does hold for some range of relevant parameters. 
Acknowledgement. (1) We would like to thank Philip Maini for providing us with the manuscript [2]; we also wish to thank him and E.A. Gaffrey for useful discussion during the preparation of the present paper.

(2) The first author is partially supported by National Science Foundation Grant DMS962287; the second author is partially supported by National Science Foundation Grant DMS 94-01251.

\section{REFERENCES}

[1] P.D. Dale, P.K Maini and J.A. Sheratt, Mathematical modeling of corneal epithelial healing, Math. Biosciences, 124 (1994), 127-147.

[2] E.A. Gaffney, P.K Maini, C.D. McCaig, M. Zhao and J.V. Forrester, Modelling corneal epithelial wound closure in the presence of physiological electric fields via a moving boundary formalism, to appear.

[3] D.A. Ladyzhenskaja, V.A. Solonnikov and N.N. Ural'ceva, Linear and Quasilinear Equations of Parabolic Type, Amer. Math. Soc., Providence, R.I. (1968).

[4] L. Olsen, P.K. Maini and J.A. Sheratt, Spatially varying equilibria of mechanical models: Application to dermal wound healing, Math. Biosciences, 147 (1998), 113-129.

[5] L. Olsen, P.K. Maini, J.A. Sheratt and B. Merchant, Simple modelling of extracellular matrix alignment in dermal wound healing I. cell flux induces alignment, to appear.

[6] L. Olsen, J.A. Sheratt, P.K. Maini and F. Arnold, A mathematical model for the capillary endothelial cell-extracellular matrix interactions in wound-healing angiogenesis, IMA J. Math. Applied to Medicine and Biology, 14 (1997), 261-281.

[7] L. Olsen, J.A. Sheratt and P.K. Maini, A mechanochemical model for adult dermal wound contraction and the permanence for the contracted tissue displacement profile, J. Theoretical Biology, 177 (1995), $113-128$. 